\title{
ADHD Medication and Substance-Related Problems
}

\author{
Robert Eme, Ph.d, A.B.P.P. \\ Illinois School of Professional Psychology at Argosy University, Schaumburg Campus \\ reme@argosy.edu
}

Attention-Deficit/Hyperactivity Disorder (ADHD), with aworld-wide prevalence in the general juvenile population of 7\% (male/female ratio of 3:1) (Owens, Cardoos, \& Hinshaw, 2015; Thomas, Sanders, Doust, Beller, \& Glasziou, 2015), is the diagnosis given to individuals who present with childhood onset of significant, impairing symptoms of inattention, impulsivity, and hyperactivity (American Psychiatric Association, 2013). These symptoms and impairments persist into adolescence and adulthood for a majority of individuals (Asherson, Buitelaar, Faraone, \& Rohde. 2016; Barkley, 2016; Hechtman, 2017).Reviews of numerous prospective studies have found that children with ADHD (combined type) are at heightened risk for substance abuse (SA) (Patrick, Foell, Venables, \& Worthy, 2016; Wilens, \& Morrison, 2015).This increased risk is reflected in prevalence rates of ADHD among adolescents and adults with substance abuse ranging from $24 \%$ to $50 \%$ (Wilens \& Morrison, 2015).Of the several possible mechanisms that might explain how ADHD increases risk for SA, the predominant mechanism involves a deviance-proneness pathway that begins with ADHDin childhood followed by oppositional/defiant behaviors, culminating in more severe conduct problem behavior (Beauchaine, Zisner, \& Sauder, 2017). It is these latter behaviors that markedly elevate the risk for developing an SA in adolescence as they not only promote risky, sensation seeking behaviors that can result in experimentation with substances of abuse but also promote associating with substance abusing peers (Molina, \& Pelham, 2014). Hence, given the risk ADHD poses for the development of SA, its successful treatment should make a significant contribution to reducing the risk of SA. However, despite the copious evidence attesting to the remarkable efficacy of short-term treatment of ADHD (e.g., up to 2 years) with stimulant medication (Connor, 2015; Hinshaw \& Ellison, 2016), there has been a long-standing concern stimulant treatment might result in an increased response to addictive substances. This concern derives from the fact that stimulant medications increase dopamine concentration in the nucleus accumbens which is a brain region implicated in SA (Koob \& Volkow, 2016) and the putative neural mechanism for ADHD treatment (Faraone et al., 2015). This concern has been addressed in two meta-analyses. A 2013 meta-analysis of 15 longitudinal studies (published between January 1980 and February 2012) with a total of 2565 individuals found that stimulant treatment for ADHD posed no increased risk for lifetime use or abuse/ dependence for alcohol, marijuana, nicotine and nonspecific drugs (Humphreys, Eng, \& Lee, 2013). A second meta-analysis in 2014 confirmed the findings for nicotine and suggested that stimulant treatment might even reduce the likelihood of cigarette smoking (Schoenfelder, Faraone, \& Kollins, 2014). However,the studies that comprise these meta-analyses suffer from a major limitation of small, non-random samples (McCabe, Dickinson, West, \& Wilens, 2016). This limitation has been overcome in three recent studies. The purpose of this brief communication is to provide the readers of this journal with a detailed review of these studies, concluding with what the best evidence-based answer is to the concern that stimulant treatment for ADHD might increase risk for SA.

\section{SWedish National Population Study}

Using Swedish national registers which provide unique personal identification numbers which enable linkage between different data bases, Chang and colleagues (2014) studied all individuals born between 1960 and 1998 and diagnosed with ADHD (26,249 men and 12,504 women). They ranged in age from 8 to 46 years, with the vast majority (79\%) being between 8 and 25 years. The study investigated the association between those who 
were exposed (16\% of males and $10.4 \%$ females) to stimulant medication (methylphenidate, amphetamine, dexamphetamine) on January 1, 2006 and any episodes of SA from the January 1, 2006 baseline to January 1, 2009. SA was indexed by substance-related death, crime, or hospital visits.Those on stimulant medication did not have increased rates of SA during the follow-up compared to those not on stimulant medication; on the contrary, the rate of SA was 31\% lower among those prescribed stimulant medication, even after controlling for numerous potential confounders (e.g., psychiatric disorder, sociodemographic measures, other psychotropic drugs such as selective serotonin reuptake inhibitor's). Also, the study found that the longer the duration of medication, the lower the rate of SA.

The two major strengths of the study are its very large population base and the use of substance-related hospitalizations, deaths, and convictions from medical and legal records to index SA without having to rely on the accuracy of respondent recall and recollection. The major limitation is that it had only a four-year interval to assess the association between stimulant medication and SA. And, as with all correlational studies, unmeasured variables cannot be ruled out as possible confounders. This limitation, which characterizes virtually every study of stimulant medication and SA, will be addressed in the paper's conclusion. Nevertheless, despite these limitations, because the study's findings are consistent with the 2 prior meta-analyses, it further strengthens the conclusion that, at the very least, stimulant treatment of ADHD does not increase the risk for SA.

\section{United States Multi-Cohort National Adolescent Study}

Nationally representative samples of high school seniors (modal age 18) in the United States drawn from 10 independent cohorts (2005-2014) were surveyed via self-administered questionnaires on whether they had ever taken any stimulant or non-stimulant medication for ADHD and substance use (McCabe et al., 2016). Use of medication for ADHD was coded for age of onset and duration of usage. Substance use was coded for usage frequency during the two weeks prior to the survey for binge drinking, past 30 days for cigarette usage, and past 12 months for marijuana and other drug use.

The study found that approximately $12.2 \%$ of the seniors had used stimulant or non-stimulant medication for ADHD in their lifetime, with $3.1 \%$ initiating stimulant treatment at age 9 years or less. Those individuals who initiated treatment at 9 years or less, or for a longer duration ( 6 or more years), had the same risk for substance use as the general population (youth without ADHD and unmedicated youth with ADHD). They also had a lower risk for substance use than those who initiated treatment later and for shorter duration. Since severity of ADHD is likely related to age of onset, duration, and use of stimulant medication (McCabe et al., 2016), and since greater ADHD severity can be expected to increase the risk for substance use, this finding is of special importance. It indicates that despite the risk severe ADHD poses for SA, early and consistent treatment with stimulant medication reduces the risk.

However, the study also found that the majority of those who imitated stimulant medication therapy for ADHD in high school reported past-year marijuana use in their senior year. The cross-sectional design of the study did not allow the determination of whether ADHD, or the short duration of stimulant treatment, increased the risk for marijuana use. The finding that a long duration of stimulant medication for ADHD (6 years or more) not only did not increase risk for SA when compared to the general population, but also reduced risk compared to a shorter duration of use in the ADHD population, would suggest that it was the ADHD rather than the stimulant medication that increased the risk for substance use. Lastly, the study found that individuals who reported only nonstimulant medication therapy for ADHD had significantly greater risk of substance use in adolescence relative to those who initiated stimulant medication therapy earlier or for longer duration. This finding may be due to the lesser effectiveness of non-stimulant medications for treating ADHD (Connor, 2015).

In conclusion, although the study has all the limitations of large-scale survey research using self-administered surveys and retrospective assessment, the consistency of its findings with the Swedish study, i.e., longer 
treatment of ADHD with stimulant medication is associated with reduced risk for substance use, attest to the accuracy of its findings. Thus, the study reinforces "the importance of early and persistent treatment of ADHD in reducing substance-related outcomes (to the level of the general population)" (McCabe et al., 2016, p.484

\section{United States Health Care Claims Study}

A replication of the Swedish study was conducted by Quinn and colleagues (2017) in the largest study to date to examine the relationship between stimulant and non-stimulant treatment for ADHD and substance-related problems. The study analyzed commercial health care claims from 2,993,887 adolescent and adult ADHD patients. Medication status was defined monthly, with a calendar month covered at least in part by a prescription considered medicated. The vast majority of prescriptions (i.e., 90\%) were for stimulant medications. Substance-related events were defined as at least one emergency department claim for non-tobacco-related substance use disorder. The findings were as follows. First, male (3.2\%) and female (2.6\%) ADHD patients were more likely to have at least one substance-related event than were controls(patients without an ADHD diagnosis), thus validating yet again the risk ADHD poses for SA. Second, relative to periods in which patients did not receive ADHD medication, males had a 33\% lower odds and females a 31\% lower odds of concurrent substance-related events when receiving medication. Moreover, males had a 19\% lower odds of substance-related events 2 years after medication periods, with female findings being less clear.Third, and most importantly, even when the results failed to support a reduction in risk (e.g., long term risk for females), the study found no evidence that medication increased risk of substance-related events, including among those with pre-existing substance use disorder. Lastly, as with the Swedish study, the study found that the results could not explained by the use of selective serotonin reuptake inhibitors.

In conclusion, as with the Swedish study, this study's two major strengths are its very large population base (indeed largest to date), and the use of formal records to index SA without having to rely on the accuracy of respondent recall and recollection. The major limitation, and to reiterate, is that as with all correlational studies, unmeasured variables cannot be ruled out as possible confounders.

\section{DISCUSSION}

The convergence of evidence from 2 meta-analyses and three recent studies that have overcome the sampling limitations of the studies in the meta-analyses strongly suggest that the concern that stimulant medication treatment for ADHD increases risk for SA is unwarranted. Indeed, there is evidence that it decreases risk for SA. The major caveat to this conclusion is that all studies that have attempted to assess the risk for posed by stimulant treatmentfor ADHD for their observational/correlational design requires the axiomatic caution that an unmeasured factor(s) might be a potential confound. Furthermore, the possibility of future research using the gold standard of random assignment to overcome this limitation is virtually impossible as it would not be feasible or ethical to assign participants to a non-treatment condition for 5 or 10 years. Nevertheless, despite the undeniable theoretical cogency of the limitation of an observational design for inferring causality, an analysis of the two most likely confounds suggests that this did not occur in the three studies that were reviewed. The first potential confound is an individual-varying selection effect in that patients who receive medication for ADHD might be different from those who don't receive medication,and this difference (which is not controlled for in the study) might produce noncausal medication associations. However, since both the Swedish and United States studies used within-individual comparisons, this ruled out all potential confounding factors that were constant within the individual over time. The McCabe and colleagues (2016) adolescent study did not employ such a design but reported a finding which would argue against the most likely selection effect, i.e., the ADHD subjects who were medicated had less severe ADHD and therefore were less likely to develop SA than the unmedicated ADHD controls. The study found just the opposite, i.e., it was the most severely affected juveniles with ADHD who were the most likely to show a protective effect from stimulant treatment. What is also remarkable about 
this finding is that it goes counter to the seemingly paradoxical finding that treatmentfor ADHD is a predictor of persistence rather than desistence of ADHD(Hechtman, 2017). This is because the most severe cases of ADHD are selected for treatment, and thus treatment is in effect a proxy for severity -a robust predictor of persistence (Hechtman, 2017). In short, the most likely selection effect in the McCabe and colleagues study (i.e., medicated patients have less severe ADHD) was not a confound.

The second most likely potential confound in a correlational/observational design is a time-varying confounding effect in that individuals receiving stimulant treatment could at the same time be receiving other treatments which could either negate the risk posed by stimulant treatment for SA or account for its protective effect for the development of SA. Two considerations argue against this possibility. First, both the Swedish and United States study ruled out the most likely treatment confound - SSRI's. Second, as noted by Quinn and colleagues(2017), studieswhichhave found that stimulant treatment of children and adults with ADHD reduced the risk of injuries and accidents are clearly less susceptible to this alternate explanation, i.e., the subjects reduced their risk for accidents because they entered some other form of treatment relevant to accident prevention concomitant with their use of medication. Therefore, if stimulant treatment alone can reduce the risk for accidents, it is likely that it also can have the same protective effect for SA, independent of whatever other treatments may have been simultaneous with the stimulant treatment.

In sum, although all the studies lacked the randomization to medication which is necessary to definitively rule out all potential confounding factors, the studies have excluded the most plausible confounds.This further reinforces the conclusion that stimulant treatment for ADHD does not increase risk for SA and may even have a protective effect. Furthermore, given that it is virtually impossible to conduct long-term studies involving randomization to medication to assess a confound-free casual effect on SA, the evidence to date is about as reliable as can be expected to address the concern that stimulant treatment of ADHD might increase risk for SA.

\section{FUTURE DIRECTIONS FOR RESEARCH}

Of the various possible future research directions, two will be presented. The first has to do with research design and the second relates to implications for treating individuals with both ADHD and SA. Regarding research design, future studies should study dosage effects and treatment trials with more than one stimulant to assess to what extent these variations may enhance a protective effect for SA. For example, one of most important findings of the largest randomized study to date of the effects of stimulant treatment of children with ADHD, the Multimodal Treatment Assessment Study, found that titrating dosage and a trial with a second different stimulant if the first treatment with a stimulant was not effective, markedly increased the rate of positive outcome in contrast to community treatment when such variations most probably were not employed (Connor, 2015).

Second, and most importantly, given the previously cited high prevalence rates of ADHD among adolescents and adults with (SA) ranging from $24 \%$ to $50 \%$ (Wilens \& Morrison, 2015), the use of stimulant medication as part of the treatment for these individuals should be vigorously explored. Only a few studies of low methodological quality with mixed results (some improvement in ADHD symptoms, but not SA) have been conducted, i.e. a "drop in the ocean" compared to what is needed (Cunill \& Castells, 2016, p.131

\section{CONCLUSION}

Three recent large-scale studies have added to the evidence base which strongly suggests that the concern that stimulant medication treatment for ADHD might increase risk for SA is unwarranted. In addition, there is also evidence that a longer treatment of ADHD with stimulant treatment might decease risk for SA. 


\section{REFERENCES}

1. American Psychiatric Association. (2013). Diagnostic and statistical manual of mental disorders (5th ed.). Washington, DC: Author.

2. Asherson, P., Buitelaar, Faraone, S., \& Rohde, L. (2016). Adult attention-deficit hyperactivity disorder: key conceptual issues. Lancet Psychiatry, 3, 568-578.

3. Barkley, R. (2016). Recent longitudinal studies of childhoodAttention-deficit hyperactivity disorder: Important themes and questions for further research. Journal of Abnormal Psychology, 125, 248-255.

4. Beauchaine, T., Zisner, A., \& Sauder, C. (2017). Trait impulsivity and the externalizing spectrum. Annual review of psychology, 13, 343-368.

5. Chang, Z., Lichtenstein, P., Halldner, L., D’Onofrio, B., Seriachius, S., Fazel, S. et al. (2014) Stimulant ADHD medication and risk of substance abuse. Journal of Child Psychology and Psychiatry, 55, 878-885.

6. Connor, D. (2015). Stimulant and nonstimulant medications for childhood ADHD. In R. Barkley (Ed.), Attention-deficit hyperactivity disorder: a handbook for diagnosis \& treatment, ( $4^{\text {th }}$ ed., pp. 666-685). New York: Guilford Press.

7. Cunill, R., \& Castells, X. (2016). The treatment of dual ADHD: a drop in the ocean. Adicciones, 28, 131-135.

8. Faraone, S., Asherson, P., Banaschewski, T., Biederman, J., Buitelaar, J., Ramos-Ouiroga, J., ...Franke, B. (2015). Attention-deficit/hyperactivity disorder. Nature Reviews: Disease Primers, 1, 1-23.

9. Hechtman L. (2017). Attention Deficit Hyperactivity Disorder: Adult outcome and its predictors. Oxford University Press.

10. Hinshaw, S., \& Ellison, K. (2016) ADHD: What everyone needs to know. New York: Oxford University Press.

11. Humphreys, K., Eng, T., \& Lee, S. (2013). Stimulant medication and substance abuse outcomes: A meta-analysis. JAMA Psychiatry, 70, 740-749.

12. Koob, G., \& Volkow, N. (2016). Neurobiology of addiction: a neurocircuitry analysis. Lancet Psychiatry, 8, 760-773.

13. McCabe, S., Dickinson, K., West, B., \& Wilens, T. (2016). Age of onset, and type of medication therapy for Attention-Deficit/Hyperactivity Disorder and substance use during adolescence: A multi-cohort national study. Journal of the American Academy of Child and Adolescent Psychiatry, 55, 479-486.

14. Molina, B., \& Pelham, W. (2014). Attention-Deficit Hyperactivity Disorder and risk of substance use disorder. Annual review of psychology, 10, 607-639.

15. Owens, E., Cardoos, S., \& Hinshaw, S. (2015). Developmental progression and gender differences in individuals with ADHD. In R.Barkley (Ed.), Attention-Deficit/Hyperactivity Disorder: A Handbook for Diagnosis and Treatment ( $4^{\text {th }}$ ed., 223-255). New York: Guilford Press.

16. Patrick, C., Foell, J., Venables, N., \& Worthy, D. (2016). Substance use disorders as an externalizing outcome. In T. Beauchaine \& S. Hinshaw (Eds.). Oxford handbook of externalizing disorders (pp. 38-60). New York: Oxford University Press.

17. Quinn, P., Chang, Z., Hur, K., Gibbons, R., Lahey, B., Rickert, M. ...D’Onofrio, B. (2017). ADHD medication and substance-related problems. American Journal of Psychiatry, Advanced on line publication, June 29. 
ADHD Medication and Substance-Related Problems

18. Schoenfelder, E., Faraone, F. \& Kollins, S. (2014). Stimulant treatment of ADHD and cigarette smoking: A meta-analysis. Pediatrics, 133, 1070-1080.

19. Thomas, R., Sanders, S., Doust, J., Beller, E., \& Glasziou, P. (2015). Prevalence ofAttention-deficit hyperactivity disorder: A systematic review of meta-analysis. Pediatrics, 135, e994-e1001.

20. Wilens, T., \& Morrison, N. (2015). Attention-deficit hyperactivity disorder and substance abuse disorder in ADHD. In L. Adler, T. Spencer, \& T. Wilens (Eds.), Attention-deficit hyperactivity disorder (pp.111-122). New York: Cambridge University Press.

Citation: Robert Eme, Ph.d, A.B.P.P. “ADHD Medication and Substance-Related Problems ”. American Research Journal of Addiction and Rehabilitation; 1(1): 10-15

Copyright (C) Robert Eme, Ph.d, A.B.P.P. This is an open access article distributed under the Creative Commons Attribution License, which permits unrestricted use, distribution, and reproduction in any medium, provided the original work is properly cited. 